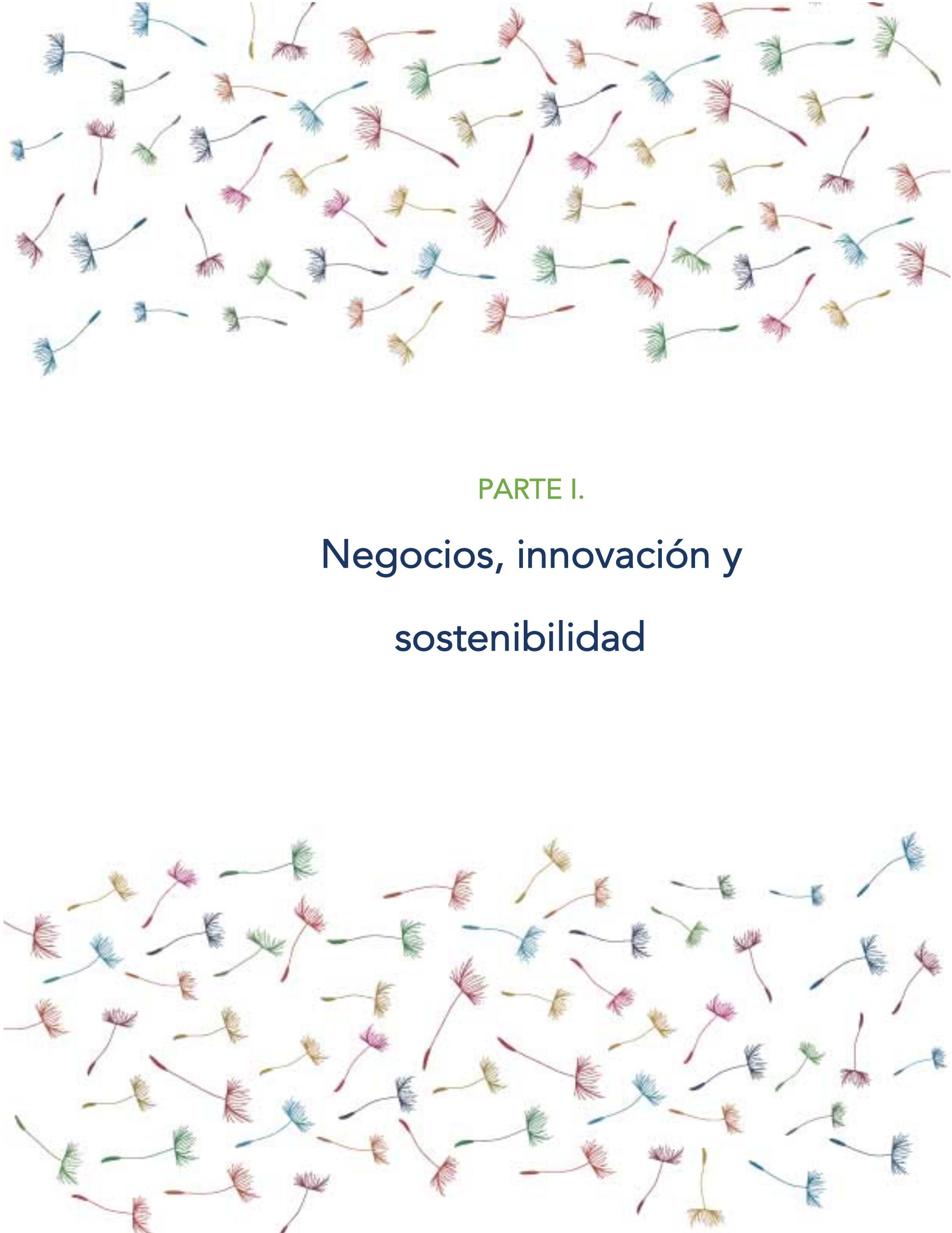




\title{
LOS CENTROS DE DISTRIBUCIÓN EN \\ PARQUES EMPRESARIALES \\ MUNICIPALES Y EL \\ OBJETIVO DE \\ DESARROLLO SOSTENIBLE $N^{\circ} 9$
}

\author{
Gonzalo Emilio León Rincón \\ gonzalo.leonri@amigo.edu.co \\ Universidad Católica Luis Amigó \\ Camilo Alberto Higuita Cárdenas \\ camilo.higuitaca@amigo.edu.co \\ Universidad Católica Luis Amigó
}

\begin{abstract}
Resumen
El presente documento aborda la investigación del impacto que está causando el desplazamiento de los centros de distribución de empresas comerciales e industriales que funcionaban al interior de la ciudad de Medellín hacia parques empresariales logísticos, ubicados en las zonas rurales de municipios cercanos, y su incidencia, principalmente en el Objetivo de Desarrollo Sostenible N9: Construir infraestructura flexible, promover la industrialización inclusiva y sostenible; y fomentar la innovación, en estos territorios. La metodología es de carácter cualitativo mediante un estudio de caso a dos empresas que se desplazaron hacia áreas rurales de municipios cercanos. Se encontró que, en los informes y planes de desarrollo, de los entes territoriales, ya están contemplados los ODS, sin embargo, no es clara la forma de lograr las metas propuestas o la forma de apalancamiento; adicionalmente, los indicadores regionales aún están en proceso de construcción. Por otro lado, la preparación logística y tecnológica de los municipios receptores de los centros de distribución aun es deficiente $y$, a diferencia de otras ubicaciones a nivel país, no están ofreciendo beneficios tributarios importantes a las empresas que decidan instalarse en los parques empresariales.

Palabras clave

Centros de Distribución, Objetivos de Desarrollo Sostenible, Plan de Ordenamiento Territorial, Logística Integral, Exenciones tributarias
\end{abstract}


Introducción

La importancia del transporte en el desarrollo sostenible fue reconocida por primera vez en la Cumbre de la Tierra de la Organización de las Naciones Unidas [ONU] en 1992, y se reforzó en el documento final Agenda 21. En la Cumbre Mundial sobre el Desarrollo Sostenible de 2002 se mencionaron diferentes puntos de apoyo para el transporte sostenible en el contexto de infraestructura, redes de entrega de bienes, sistemas de transporte público, asequibilidad, eficiencia y conveniencia del transporte, así como también la necesidad de mejorar la calidad y la salud del aire urbano, y reducir las emisiones de gases de efecto invernadero (ONU, 2002).

En Colombia, el resto de las etapas de la cadena logística han ayudado a dinamizar y contribuir en el diseño de estrategias que inciden en el logro de los objetivos propuestos por la Organización de Naciones Unidas Conocido como Objetivos de Desarrollo sostenible [ODS], en especial desde el Objetivo $N^{\circ} 9$ Construir infraestructura flexible, promover la industrialización inclusiva y sostenible; y fomentar la innovación.

En la presente investigación se pretende documentar cuáles son los impactos que tiene el sector logístico, sobre los Objetivos de Desarrollo Sostenible regionales o locales, tomado como base las tendencias del mercado, los diferentes marcos regulatorios, los planes de desarrollo local y contrastarlos con una investigación de campo en empresas que decidieron ubicar sus centros de distribución [CEDIS] en las áreas rurales -conocidos como Parques Industriales- de municipios cercanos a la capital del departamento de Antioquia. 
Cabe resaltar que los ODS no son vinculantes para ningún país, de forma jurídica, sin embargo los gobiernos participantes de la Agenda de Acción de Addis Abeba de la Tercera Conferencia Internacional sobre la Financiación para el Desarrollo- (ONU, 2015a), se comprometieron a adoptar como propios, los objetivos propuestos y establecer prioridades y regulaciones que permitan alcanzar las metas fijadas para el año 2030 -Agenda 2030- mediante planes y propuestas de desarrollo, compromiso refrendado con la firma de la resolución A/Res/69/313 en Nueva York (ONU, 2015b).

De acuerdo con el Banco Interamericano de Desarrollo [BID] los ODS esenciales son "poner fin a la pobreza en todas sus formas, reducir la desigualdad, global, y al interior de las sociedades nacionales, luchar contra el cambio climático, garantizando, al mismo tiempo, que nadie [ningún habitante del planeta] se quede atrás". (Carlino, Netto, Suarez y Vasa, 2017, p. 7) Sin embargo la puesta en marcha del Objetivo $N^{\circ} 9$ se puede considerar como primordial para poder dar cumplimiento a estos primeros objetivos básicos.

Del lado empresarial la globalización ha impulsado el desarrollo de nuevos modelos de negocio en el cual la logística se ha convertido en el diferencial más importante, puesto que el valor agregado esperado por los clientes, solo es percibido a través de cadenas de suministro bien administradas. La logística como herramienta de gestión indispensable para apalancar el crecimiento de la organización demanda grandes inversiones en temas como el almacenamiento, el transporte y la distribución, los cuales se ven afectados en las grandes ciudades por temas como la orientación y el uso de la tierra, la gestión 
vehicular y las restricciones que las políticas locales que cada ciudad pueda aplicar a los vehículos de transporte de carga.

Notteboom y Rodríguez (2005), encontraron que la globalización madura ha hecho que la especialización local en logística industrial sea más marcada, empujando a las empresas a concentrarse en lugares con ventajas específicas, como centros logísticos, puertos secos y parques logísticos o empresariales. En la actualidad, a nivel mundial se puede observar una gran demanda de nuevas instalaciones logísticas y CEDIS, como lo documenta Majchrzak-Lepczyk (2017), quien afirma que para finales de 2015 ya había cerca de 19.8 millones de metros cuadrados en CEDIS entre Europa Central y Oriental. En las últimas décadas, el modelo industrial de la Unión Europea se ha basado en redes periféricas y en el uso generalizado de almacenes, puntos de tránsito y depósitos. Esto es causado por la globalización de la producción, la integración de países, así como la reestructuración y la tercerización en el mercado logístico (McKinnon, 2009).

Al igual que en las grandes ciudades del mundo, en el Valle de Aburrá, Antioquia, ha venido ocurriendo un fenómeno importante en cuanto al uso de la tierra. Las Industrias que a mediados del siglo pasado construyeron sus sedes en la periferia de los municipios, hoy se encuentran rodeadas de viviendas residenciales que les impiden expandirse y por el contrario reclaman esos espacios en pro del bienestar ambiental ya mencionado y que impactan directamente en el espacio reclamado por nuevas familias ya sea conformadas localmente o migrantes de áreas rurales o suburbanas. Es así como los centros industriales y las fábricas de la ciudad han venido desplazando sus 
almacenamientos y CEDIS hacia las zonas rurales de los pueblos cercanos, modificando también los indicadores propios de cada una de estas regiones, probablemente para algunos de estos municipios, de manera sorpresiva.

En contraste, y aunque en Colombia la normatividad aún tiene falencias que aseguren la distribución equitativa de la propiedad rural $y$, que los sistemas de información de catastro y registro se encuentran desactualizados (Rojas B, 2015), las administraciones nacionales, regionales y municipales han venido modificando el uso de la tierra, a partir de consideraciones como el desplazamiento o migración de los habitantes de zonas rurales hacia las áreas urbanas metropolitanas. A nivel mundial se prevé que para el año 2050, el desplazamiento, esté por encima de un 66\% (Wilmsmeier, 2015). En Colombia, el Congreso aprobó la Ley 1900 (de 18 Junio, 2018) -Ley de tierras-, que incorpora parte de los acuerdos en materia de sustitución y restitución de tierras, suscritos con la cúpula de la antigua guerrilla de las FARC.

En Medellín, desde el 16 de Diciembre de 2014 se encuentra vigente un plan de ordenamiento territorial [POT], el cual dentro de sus lineamientos incluye la construcción de más de 400 kilómetros de ciclo-ruta, 500 pasos peatonales, mejorar el transporte público, implementar vías de tráfico calmado, frenar la expansión en las laderas, densificar la ciudad hacia el centro, controlar zonas de alto riesgo, disminuir el déficit de vivienda y mejorar los barrios que están atrasados (Acuerdo 48, 2014). Adicionalmente, en diciembre de 2019 se adoptó el Plan Estratégico Metropolitano de Ordenamiento Territorial del Valle de Aburrá, el cual será el marco al cual deberán 
acogerse cada uno de los municipios que conforman el área, al adoptar los planes básicos de ordenamiento territorial (Acuerdo Metropolitano $N^{\circ}$ 31, 2019).

En esta, la era de la globalización, la actividad económica es dinámica y en permanente evolución, los servicios logísticos, de infraestructura y los servicios de movilidad también forman parte integral de esta, y al mismo tiempo son facilitadores del desarrollo económico y social. Adicionalmente es importante resaltar que en la actualidad democrática encontramos una gran variedad de modelos de gobierno y regulaciones de Ley que influyen sobre estos tres sistemas (Wilmsmeier, 2015). Las regulaciones adicionales a los POT, que podrían influir en la ubicación de los CEDIS, tienen que ver con atractivos económicos, financieros o tributarios que los gobiernos nacionales y/o locales puedan ofrecerles a los empresarios, para lo cual revisaremos las leyes que más inciden en estos aspectos y las decisiones que algunos municipios han tomado para atraer a los empresarios a instalarse en diferentes parques industriales ubicados en cada territorio.

Se pretende entonces con esta investigación indagar el impacto generado por la localización y construcción de los nuevos CEDIS, y su incidencia en los ODS regionales $\mathrm{N}^{\circ} 9$.

El documento aborda los marcos de trabajo para el ODS $\mathrm{N}^{\circ} 9$, los componentes económicos para la puesta en marcha de los planes de desarrollo municipal, las reglamentaciones para los parques industriales y logísticos, teniendo en cuenta los atractivos, beneficios y regulaciones.

En la sección de análisis se documentan los hallazgos de las entrevistas a los representantes de las empresas usuarias de estos parques y se 
OBJETIVOS DE DESARROLLO SOSTENIBLE

Avances y experiencias hacia su cumplimiento

contrastan con el estado de los municipios que los acoge, así como su impacto en los ODS regionales.

Los ODS en Colombia

Como se mencionó en la introducción, para la presente investigación nos centraremos en revisar el alcance del Objetivo Nº puesto que a partir de este, se podrá dar respuesta a los objetivos esenciales enunciados la Tercera Conferencia Internacional sobre la Financiación para el Desarrollo (2015), en la cual los países firmantes dijeron:

\footnotetext{
Nosotros, los Jefes de Estado y de Gobierno y Altos Representantes, reunidos en Addis Abeba del 13 al 16 de julio de 2015, afirmamos nuestro fuerte compromiso político para abordar el desafío de la financiación y la creación de un entorno propicio a todos los niveles para el desarrollo sostenible, en el espíritu de asociación global y solidaridad. Reafirmamos y construimos sobre el Consenso de Monterrey de 2002 y la Declaración de Doha de 2008. Nuestro objetivo es acabar con la pobreza y el hambre y alcanzar el desarrollo sostenible en sus tres dimensiones mediante la promoción del crecimiento económico inclusivo, la protección del medio ambiente y la inclusión social. Nos comprometemos a respetar todos los Derechos Humanos, incluido el Derecho al Desarrollo. Aseguraremos la igualdad de género y el empoderamiento de las mujeres y las niñas. Promoveremos sociedades pacíficas e inclusivas y avanzaremos plenamente hacia un sistema económico global equitativo en el que ningún país o persona se quede atrás, permitiendo un trabajo decente y medios de vida productivos para todos, mientras preservamos el planeta para nuestros niños y las generaciones futuras. (ONU, 2015a, p. 1)
}

El Objetivo N 9, Industria, Innovación e Infraestructuras propone cinco acciones orientadas al fortalecimiento de la infraestructura básica, tanto regional como transfronteriza, a promover el desarrollo industrial de la pequeña y gran industria garantizando el apoyo 
financiero y su integración con las cadenas de valor y en la Infraestructura Tecnológica, y a incrementar el acceso a las Tecnologías de Información y comunicación promoviendo a su vez la investigación científica y tecnológica en todos los niveles (ONU, 2016).

Si revisamos las propuestas de este Objetivo, se puede inferir que al dinamizar la Industria y mejorar la Infraestructura, necesariamente hay generación de empleo tanto de mano de obra básica como especializada y, al promover la Investigación científica, se podrán implementar procesos que agreguen valor. Estas acciones incidirán positivamente y de manera directa en los ODS uno a cuatro e indirectamente en los demás.

El Gobierno Colombiano para dar cumplimiento a la Agenda 2030, en la cual, a partir de los compromisos en los ODS y los resultados obtenidos en los Objetivos del Milenio [ODM], estableció una hoja de ruta, la cual publicó en el documento CONPES 3918 -Estrategia Para La Implementación De Los Objetivos De Desarrollo Sostenible (ODS) En Colombia- (Departamento Nacional de Planeación [DNP], 2018), el cual recomienda que cada región incorpore, desde su formulación, planes de desarrollo que incluyan iniciativas de ODS acordes con los esfuerzos regionales requeridos para reducir las disparidades territoriales; y seleccionar ponderadores idóneos y pertinentes para cada ODS, en las que se tengan en cuenta de manera particular para cada región, entre otros: la población total, la población rural, la población económicamente activa, hogares urbanos, viviendas y PIB departamental. También le encomendó a la Escuela Superior de Administración Pública [ESAP] brindar capacitaciones a los candidatos a las alcaldías y gobernaciones para la adecuada apropiación de los ODS y su inclusión en la definición 
de programas de gobierno y en los procesos de planeación territorial y planes Básicos de Ordenamiento Territorial, para los nuevos períodos (DNP, 2018).

Para este objetivo se tienen actividades previas, plasmadas en otros documentos CONPES que promueven el desarrollo y la infraestructura, entre ellos el 3547 -Política Nacional Logística- (DNP, 2008), a partir del cual se aprobaron las vías 4G, la masificación del Internet y la modernización tecnológica de las Zonas francas y Aduanas nacionales y aprobación de puertos secos, entre otros. El CONPES 3457 de 2007, 3032 de 1999, 3072 de 2000 y 3171 de 2002, los cuales fueron publicados para cumplir con objetivos tales como llevar las Tecnologías de Información y Comunicaciones [TIC], a los lugares más apartados y/o desatendidos tales como escuelas, viviendas urbanas y rurales de estratos 1 y 2; y fortalecer la presencia virtual de las empresas e Instituciones Públicas a través de Conectividad en banda ancha y promover la iniciativa Colombia Digital.

Estos documentos han sido reforzados por los recientes CONPES 3982 -Política Nacional Logística- cuyo objetivo Principal es "Promover la intermodalidad en el transporte y la facilitación del comercio, para reducir los costos y tiempos logísticos; y así, impulsar la competitividad del país" (DNP, 2020, p. 75) y el CONPES 3975 -Política Nacional Para la Transformación Digital e Inteligencia Artificial- con el cual se pretende:

Aumentar la generación de valor social y económico a través de la transformación digital del sector público y del sector privado, mediante la disminución de barreras, el fortalecimiento del capital humano y el desarrollo de condiciones habilitantes, para que Colombia pueda aprovechar las oportunidades y enfrentar los retos 
Para poner en funcionamiento cualquier iniciativa que suponga erogaciones, los entes territoriales deben contar con recursos. De acuerdo con el Departamento Nacional de Planeación [DNP], para el año 2015 los municipios en Colombia recibieron recursos de diferentes fuentes cuya composición se aprecia en la Gráfica 1.

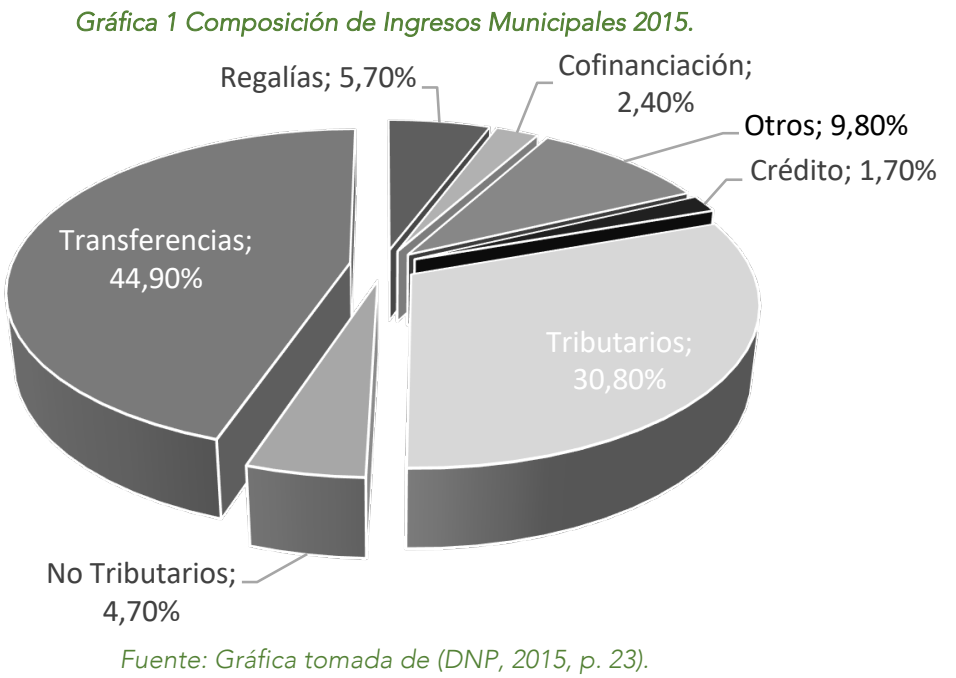

En la Gráfica 1 se aprecia que los rubros transferidos desde el Gobierno Nacional, en especial los recursos del Sistema General de Participación, es la porción mayor para la inversión y gastos propios de cada municipio (DNP, 2015). Quiere decir esto que si el Municipio quisiera apalancar o fortalecer iniciativas de acción social -ODS, por ejemplo-, y las transferencias nacionales no son suficientes, deberá buscar alternativas de financiación, o presentar proyectos bien estructurados 
para obtener fondos de cofinanciación por un lado o incrementar el rubro de ingresos tributarios.

Es importante resaltar que, en materia de ingresos tributarios, la Constitución Política de Colombia (1991), en su Artículo 294 define que "La ley no podrá conceder exenciones ni tratamientos preferenciales en relación con los tributos de propiedad de las entidades territoriales. Tampoco podrá imponer recargos sobre sus impuestos salvo lo dispuesto en el artículo 317". Adicionalmente, y de acuerdo con el Artículo 38 de la Ley 14 (1983) "el municipio solo podrá otorgar exenciones tributarias por un plazo limitado, que en ningún caso excederá de diez (10) años, de conformidad con el plan de desarrollo municipal. La exención no podrá ser solicitada con retroactividad" (Sánchez-Cubides, 2014, p. 165).

Para entender mejor de donde provienen las fuentes de financiación de los entes municipales, se presenta en la Tabla 1 la clasificación de estos ingresos.

Al hacer una revisión cuidadosa de los rubros, para apalancar los Planes y Proyectos de Gobierno municipal, se deben revisar aquellos que pudieran ser modificados, o negociados a largo plazo y que permitan el incremento de los ingresos corrientes o revisar el CONPES 2791 -Ajustes al Sistema Nacional de Cofinanciación- y sus leyes reglamentarias que permitan presentar proyectos que puedan ser cofinanciados. 
OBJETIVOS DE DESARROLLO SOSTENIBLE

Avances y experiencias hacia su cumplimiento

Tabla 1 Clasificación de los Ingresos en Municipios Colombianos

\begin{tabular}{|c|c|c|c|c|}
\hline \multicolumn{2}{|c|}{$\begin{array}{c}\text { CLASIFICACIÓN DEL } \\
\text { INGRESO }\end{array}$} & \multirow[b]{2}{*}{$\begin{array}{l}\text { Contribución obligatoria } \\
\text { causada por un hecho de } \\
\text { naturaleza jurídica, exigida sin } \\
\text { contraprestación alguna, que } \\
\text { recae sobre personas naturales } \\
\text { o jurídicas, }\end{array}$} & \multicolumn{2}{|c|}{ RUBROS } \\
\hline \multirow{3}{*}{$\begin{array}{l}\text { Ingresos } \\
\text { corrientes }\end{array}$} & Tributarios & & $\begin{array}{l}\text { - Predial } \\
\text { - Impuesto vehículos } \\
\text { automotores } \\
\text { - Industria y comercio } \\
\text { - Avisos y tableros } \\
\text { - Espectáculos públicos } \\
\text { - Degüello ganado menor } \\
\text { - Rifas municipales } \\
\text { - Estampillas }\end{array}$ & $\begin{array}{l}\text { - Juegos permitidos } \\
\text { - Publicidad exterior visual } \\
\text { - Delineación y urbanismo } \\
\text { - Sobretasa gasolina } \\
\text { - Iva turístico } \\
\text { - Contribución } 5 \% \\
\text { contratos sobre obra } \\
\text { pública } \\
\text { - Impuesto de } \\
\text { hidrocarburos }\end{array}$ \\
\hline & $\begin{array}{c}\text { No } \\
\text { Tributarios }\end{array}$ & $\begin{array}{l}\text { Se originan por la prestación de } \\
\text { un servicio público, la } \\
\text { explotación de bienes o la } \\
\text { participación de beneficios de } \\
\text { bienes o servicios. }\end{array}$ & $\begin{array}{l}\text { - Acueducto } \\
\text { - Alcantarillado } \\
\text { - Matadero } \\
\text { - Alumbrado público } \\
\text { - Energía eléctrica } \\
\text { - Telefonía } \\
\text { - Servicio aseo } \\
\text { - Plaza de mercado }\end{array}$ & $\begin{array}{l}\text { - Nomenclatura } \\
\text { - Registros industria y } \\
\text { comercio } \\
\text { - Marcas y herretes } \\
\text { - Arrendamientos } \\
\text { - Multas de gobierno } \\
\text { - Multas de planeación } \\
\text { - Multas de rentas } \\
\text { - Multas de tránsito }\end{array}$ \\
\hline & $\begin{array}{c}\text { Transferencia } \\
\text { s }\end{array}$ & $\begin{array}{c}\text { Recursos financieros que se } \\
\text { trasladan de un ente estatal a } \\
\text { otro. }\end{array}$ & $\begin{array}{l}\text { - Las transferencias de la } \\
\text { Nación }\end{array}$ & $\begin{array}{l}\text { - Sistema General de } \\
\text { Participaciones SGP }\end{array}$ \\
\hline \multirow{4}{*}{$\begin{array}{l}\text { Ingresos de } \\
\text { Capital }\end{array}$} & Crédito & $\begin{array}{l}\text { Son los ingresos provenientes } \\
\text { de empréstitos o colocaciones } \\
\text { de papeles en el mercado } \\
\text { financiero, con vencimiento } \\
\text { mayor a un año, concedidos } \\
\text { directamente a los entes } \\
\text { territoriales, }\end{array}$ & $\begin{array}{l}\text { - Créditos internos: } \\
\text { Findeter, Institutos de } \\
\text { Desarrollo Departamental, } \\
\text { Fonade }\end{array}$ & $\begin{array}{l}\text { - Créditos internacionales } \\
\text { del nivel central: BIRF, BID }\end{array}$ \\
\hline & Regalías & $\begin{array}{l}\text { Compensación por el impacto } \\
\text { que causa la explotación de } \\
\text { recursos no renovables y el } \\
\text { agotamiento de los mismos. }\end{array}$ & $\begin{array}{l}\text { - Minería } \\
\text { - Petróleo }\end{array}$ & - Generación de Energía \\
\hline & $\begin{array}{c}\text { Cofinanciació } \\
n\end{array}$ & $\begin{array}{l}\text { Recursos condicionados, } \\
\text { orientados por la Nación hacia } \\
\text { sectores y programas prioritarios } \\
\text { del Plan Nacional de Desarrollo, } \\
\text { focalizando, el gasto hacia las } \\
\text { poblaciones más vulnerables, } \\
\text { mediante la presentación de } \\
\text { proyectos }\end{array}$ & - Propuestas de ejecución c & e proyectos especiales \\
\hline & Otros & $\begin{array}{c}\text { Recursos carentes de } \\
\text { clasificación en cuanto a } \\
\text { Ingresos Corrientes o de Capital }\end{array}$ & $\begin{array}{l}\text { - Donaciones } \\
\text { - Excedentes financieros } \\
\text { de establecimientos } \\
\text { públicos }\end{array}$ & $\begin{array}{l}\text { - Venta de activos } \\
\text { - Bonos }\end{array}$ \\
\hline
\end{tabular}


De los parques industriales y su reglamentación

De acuerdo con las Directrices Internacionales para Parques Industriales publicada por la ONU (2019), un parque industrial es "una extensión de tierra desarrollada y subdividida en parcelas de acuerdo con un plan integral, aprovisionado con carreteras, transporte y servicios públicos, a veces también con instalaciones comunes, para el uso de un grupo de empresas" (p. 26). Sin embargo, el término parques industriales también se usa a menudo para cubrir una amplia gama de conceptos, tales como zonas de libre comercio, zonas de procesamiento de exportaciones, zonas económicas especiales, zonas de alta tecnología, puertos libres, zonas empresariales, entre otros.

El primer parque industrial fue Trafford Park, construido para una compañía llamada Shipcanal and Docks cerca de Manchester en 1896. El surgimiento masivo de los parques industriales fue motivado por conceptos de políticas regionales, que pretendían paliar la crisis de Gran Bretaña en la década de 1930 (ONU, 2019). En 1960 ya existían 46 en el Reino Unido y su concepto y construcción se fue expandiendo por toda Europa de manera paulatina. En 1984, Alemania Occidental contaba con 22 parques (Vidova, 2010). En Estados Unidos fue solo después de la Segunda Guerra Mundial que tomaron fuerza y popularidad (Phillimore \& Joseph, 2003).

De acuerdo con la ONU (2019), los parques industriales se construyeron tradicionalmente fuera de los límites de la ciudad debido a los menores costos de la tierra, con el ánimo de evitar 
incompatibilidades con áreas residenciales y comerciales; y la capacidad de gestionar temas ambientales. Se pueden encontrar cuatro generaciones, la primera generación se construyó comenzando la década de 1970 y se distinguieron por tener una arquitectura bastante simple, salas de reunión, almacenes y un área administrativa que ocupaba solo del 10\% al 15\% del total del parque. La segunda generación se construyó entre 1975 y 1985, y sus oficinas, que fueron utilizadas por empresas dedicadas a la ciencia, la tecnología y los negocios, ocuparon un espacio mucho mayor, su característica principal fue una arquitectura desafiante y más compleja. Desde la segunda mitad de la década de 1980, se construyeron los parques industriales de tercera generación; estos fueron típicos por el uso elástico del área y una amplia cartera de servicios, así como por un aumento en el número de personal administrativo $y$, además, con un espacio más amplio para las áreas de Tecnología Informática. La cuarta generación se caracteriza por tener grandes edificios administrativos y un amplio portafolio de servicios, los surgieron a partir de mediados de los años noventa. Desde la segunda mitad de la década de 1990, los parques industriales han sido parte de una red internacional de Parques Industriales.

En Colombia, desde 1950, el Gobierno había publicado el decreto 384 el cual fomentaba la concentración de bodegas para realizar actividades de recepción, almacenamiento y distribución en lo que originalmente se denominó Urbanizaciones Industriales. En 1974, en México, se llevó a cabo la Conferencia Latinoamericana de Industrialización. En esta, los gobiernos se comprometieron a incrementar su participación en la industria manufacturera mundial, para pasar de un $5 \%$ de ese entonces al 
13.5\% en el año 2000. Con base en estos compromisos, el Estado promulgó leyes y decretos adicionales que le terminaron de dar forma a los Parques Industriales y Zonas Francas a saber: En 1976 con el decreto 2613 se impulsó la creación de siete nuevos parques industriales en Pereira, Barranquilla, Cúcuta, Bucaramanga, y Quindío, adicionales a los ya existentes en las ciudades de Duitama y Sogamoso y, en 1979, con la aprobación y publicación del decreto 2143, se perfeccionó el concepto, sus reglamentaciones y medidas de estímulo y en el decreto 2233 de 1996 le dio cuerpo a las Zonas Francas (Leal, 2005).

De acuerdo con Monroy y Ramírez (2003), el concepto de Parque Industrial Eco-eficiente hizo su aparición en 1997, aunque solo después del Año 2000 comenzó a tomar cuerpo, con la publicación de los primeros POT y los componentes de actividades e indicadores del término Ecoeficiente, que incluía: el consumo de agua, energía eléctrica, disposición de desperdicios y basuras y emisión de gases, entre otros.

Vale mencionar que La ley 218 de 1995 conocida como Ley Páez, otorga beneficios interesantes a empresas y Parques Industriales, aunque su cobertura es en un área geográfica determinada. A raíz un sismo de gran intensidad, con epicentro en cercanías del Municipio de Toribío en el Departamento del Cauca el 6 de junio de 1994, el Gobierno Nacional declaró la emergencia económica y buscando dinamizar la economía de los lugares afectados promulgó esta ley, la cual, junto con sus decretos reglamentarios 0529 y 2340 de 1996, estableció exenciones para las empresas que se fundarán o se 
trasladarán a los municipios o áreas de los municipios afectados. Esta ley tuvo modificaciones en el decreto 529 de 1996 y la ley 488 de 1998 que en su Artículo 26 extendió los beneficios tributarios de la ley a entidades territoriales que se formen dentro de los límites territoriales originales mencionados (DIAN, 2000).

\section{Exenciones Tributarias}

El artículo 32 de la Ley 14 (1983), en relación con el impuesto de Industria y Comercio dice:

El Impuesto de Industria y Comercio recaerá, en cuanto a materia imponible, sobre todas las actividades comerciales, industriales y de servicio que ejerzan o realicen en las respectivas jurisdicciones municipales, directa o indirectamente, por personas naturales, jurídicas o por sociedades de hecho, ya sea que se cumplan en forma permanente $u$ ocasional, en inmuebles determinados, con establecimientos de comercio o sin ellos.

El artículo 59 de la Ley 788 (2002) dispone:

Procedimiento tributario territorial. Los departamentos y municipios aplicarán los procedimientos establecidos en el Estatuto Tributario Nacional, para la administración, determinación, discusión, cobro, devoluciones, régimen sancionatorio incluida su imposición, a los impuestos por ellos administrados. Así mismo aplicarán el procedimiento administrativo de cobro a las multas, derechos y demás recursos territoriales. El monto de las sanciones y el término de la aplicación de los procedimientos anteriores, podrán disminuirse y simplificarse acorde con la naturaleza de sus tributos, y teniendo en cuenta la proporcionalidad de estas respecto del monto de los impuestos.

Estos dos artículos de ley facultan entonces a las autoridades municipales para administrar los impuestos y establecer las exenciones para aquellos parques industriales o logísticos cuya clasificación sea 
diferente a Zonas Francas, las cuales gozan del tratamiento tributario dispuesto en las leyes de Parques Industriales

\section{Metodología}

La metodología empleada es de carácter cualitativo, con un alcance exploratorio descriptivo, basada en estudios de caso de dos organizaciones colombianas que decidieron reubicar sus CEDIS, una en un municipio del extremo Norte del Valle de Aburrá y la otra en otro municipio, del extremo sur, cerca de corredores viales estratégicos y construidos en lo que se conoce hoy como parques industriales.

El muestreo realizado es no probabilístico por conveniencia. La selección se realizó a partir de una lista de 34 empresas que se han desplazado a parques industriales. Se confirmó que los entrevistados fueran los responsables directos, o tuvieran acceso a la información concerniente al proceso de selección, estudios de diseño, e implementación de los CEDIS, quienes conocen de primera mano las ventajas y beneficios percibidos, así como los tropiezos y dificultades presentadas y también de los impactos generados por la ubicación de estos CEDIS en dichos municipios. La recolección de la información se llevó a cabo durante mayo y junio de 2020.

La información primaria se obtuvo a partir del diseño de un formulario que sirvió de base para las entrevistas en profundidad, dividido en cinco secciones. La primera sección aborda las generalidades de organización, la segunda indaga sobre los procesos 
logísticos que se llevan a cabo en el CEDI, la tercera busca confirmar cuales fueron los elementos claves que se tuvieron en cuenta para la elección de la ubicación y la cuarta busca documentar las ventajas y beneficios percibidos para la organización y el municipio, así como los impactos generados en aspectos sociales, económicos y logísticos.

Las organizaciones que participaron en la investigación fueron la Industria de Aseo y Cuidado personal cuya sede principal está ubicada en el departamento de Antioquia, y la segunda es del sector de Alimentos cuya sede principal está en el Valle del Cauca. Las dos tienen presencia nacional e internacional y que de acuerdo con la Ley de Habeas Data pidieron mantener su nombre en reserva.

También se obtuvo información a partir de fuentes secundarias mediante la revisión detallada de repositorios institucionales, bases de datos, archivos, informes y revistas especializadas en el área.

Con la información recabada, se procedió a tabular los datos. Se enumeraron los hallazgos más importantes y coincidentes en la implementación de estos CEDIS y sus impactos en los municipios.

Entre las limitaciones en el proceso de investigación se encuentra la disponibilidad de otras empresas que cuentan con similares características en cuanto a la reubicación de sus CEDIS, puesto que sus actividades estaban concentradas en el diseño de planes logísticos que incluyeran la Bioseguridad y a la vez los ajustes de los procesos a partir de los impactos económicos causados por las restricciones del comercio y movilidad en respuesta a la crisis generada por la pandemia del COVID19, por lo cual respetuosamente declinaron hacer parte de la investigación. 
El tipo de investigación es descriptivo, cualitativo y analítico debido a que se busca identificar las diferentes motivaciones y resultados obtenidos en el diseño construcción y puesta en marcha de centros de distribución por fuera de Medellín en empresas con sede en el municipio.

\section{Discusión y resultados}

\section{Situación Productiva y Económica de Colombia}

De acuerdo con Ramírez e Higuera (2018), en Colombia durante los tres primeros lustros del Siglo $\mathrm{XXI}$, hubo en promedio un incremento en el sector de la economía de $4.8 \%$ por año y la inversión tuvo un incremento de un $26 \%$ en el 2014 frente al $13 \%$ en el año 2000. Con la situación actual de crisis (pandemia Covid-19), será algo complejo encontrar, en el futuro cercano, índices de crecimiento como los mencionados. Adicionalmente revisando los componentes estructurales del PIB, que se presentan en la Tabla 2, durante ese mismo período la industria manufacturera tiene una tendencia decreciente muy marcada. Tendencia que podría afectar mucho más el logro de los ODS.

Tabla 2. Composición de PIB a partir de los sectores productivos en Colombia

\begin{tabular}{cccc}
\hline Sector & 2000 & 2006 & 2014 \\
\hline Agricultura, ganadería, caza, silvicultura y pesca & $8,7 \%$ & $8,1 \%$ & $6,8 \%$ \\
Explotación de minas y canteras & $8,7 \%$ & $6,6 \%$ & $8,1 \%$ \\
Industrias manufactureras & $14,8 \%$ & $15,5 \%$ & $12,3 \%$ \\
Suministro de electricidad, gas y agua & $4,5 \%$ & $4,3 \%$ & $3,8 \%$ \\
Construcción & $4,8 \%$ & $6,4 \%$ & $7,9 \%$ \\
Comercio, reparación, restaurantes y hoteles & $12,7 \%$ & $13,2 \%$ & $13,3 \%$ \\
Transporte, almacenamiento y comunicaciones & $6,9 \%$ & $7,8 \%$ & $8,0 \%$
\end{tabular}


Establecimientos financieros, seguros, actividades $\quad 21,0 \% \quad 20,9 \% \quad 21,8 \%$ inmobiliarias y servicios a las empresas

En referencia a las cifras de empleo, Ramírez e Higuera (2018), sostienen que este está concentrado en sectores con bajos niveles de productividad, mientras en los sectores de alta productividad como los mineros, eléctricos y de gas presentan una disminución ostensible de participación. Adicionalmente, frente a los demás países pertenecientes a la OCDE los niveles de productividad son muy inferiores; y a la vez, también lo son frente al promedio de Latinoamérica.

En cuanto las exportaciones, los primarios son los que van ganando cada vez mayor participación, mientras que los elementos de tecnología que en algún momento alcanzaron el 30\%, en 2014 se habían reducido al 10\% (Ramírez \& Higuera, 2018).

El panorama para Antioquia, con datos presentados hasta 2019, tenía tendencias más halagadoras, pues de acuerdo con la Cámara de Comercio de Medellín para Antioquia, había mejorado la confianza del consumidor en $1.1 \%$; el crecimiento en ventas minoristas era del $3.7 \%$, las exportaciones habían crecido en $4.5 \%$ y el de constitución de empresas en 7.5\% (Cámara de Comercio de Medellín, 2019).

Para Medellín los indicadores tenían también tendencias positivas puesto que las cifras hasta junio de 2019 incluían crecimientos en ventas de $6.1 \%$ frente al mismo periodo del año anterior, al igual que en producción cuyo incremento fue del $4.7 \%$ y del $0.6 \%$ en la generación de empleo (Cámara de Comercio de Medellín, 2019). 
Los ODS en Colombia - resultados

Los resultados de los ODS tienen un primer informe voluntario realizado por la Presidencia de la Republica con fecha 1 de julio del 2018 en el cual se analizan las acciones de los años 2016 y 2017 teniendo en cuenta que, de acuerdo con la hoja de ruta, todavía se encuentra en la etapa de conformación de Indicadores finales para los 16 objetivos. En el documento se encuentran algunos resultados a partir de proyectos pilotos (Secretaría Técnica de la Comisión ODS, 2018).

En el área de inversión se destaca que los recursos para los ODS se redujeron aproximadamente en un 11\% entre el 2015 y 2017 principalmente ocasionados por la caída de los precios del petróleo. Para el objetivo 9 -de nuestro interés-, los recursos invertidos tuvieron una participación del $10.3 \%$ principalmente aportados por el presupuesto del Gobierno Nacional y del Sistema General de Regalías. En el informe presentado, aun no se registra indicador que mida el cumplimiento frente a la meta para el ODS 9 (Secretaría Técnica de la Comisión ODS, 2018).

Los resultados de los ODS para Antioquia se buscaron en la página en línea, sin embargo, solo se encuentra información completa del objetivo número 5 y ningún tipo de información para los ODS 9, y 11 a 17. $\operatorname{Los} \operatorname{ODS} 1,2,3,4,6,8$, y 10 contienen indicadores parciales. 
Los planes de desarrollo municipales

Los planes de desarrollo para el período de 2020 a 2023 que fueron publicados por las administraciones municipales de Caldas y de Girardota incluyen los lineamientos y propuestas para dar cumplimiento a los ODS.

Las acciones principales para el ODS $N^{\circ} 9$ en el municipio de Caldas están orientadas al fortalecimiento de la red vial del municipio, y la construcción y mejoramiento de espacios públicos integrales y nuevos espacios urbanos. Extrañamente no se menciona ningún interés en los centros producción logística ubicados largo de la vía Troncal y su interconexión con transporte público a la Zona Franca Centralidad Metropolitana Primavera (Consejo Municipal de Caldas, 2020).

En el Plan de Desarrollo del municipio de Girardota, para él ODS N9, las propuestas presentadas en están orientadas al emprendimiento sostenible y al fortalecimiento empresarial, buscando empresas de economía circular, iniciativas de emprendimiento apoyadas bajo el modelo de economía solidaria; y a nivel tecnológico, el proyecto macro corresponde a la puesta en marcha de una plataforma web inteligente, para articular procesos de vinculación laboral entre las empresas existentes y los habitantes del municipio (Consejo Municipal de Girardota, 2020). 
En ausencia de los presupuestos en los planes de desarrollo publicados, las asignaciones presupuestales, fueron consultadas en la página del DNP. Estas y su distribución se muestran en la Gráfica 2.

Gráfica 2. Distribución porcentual de asignaciones SGP por sectores en los dos municipios
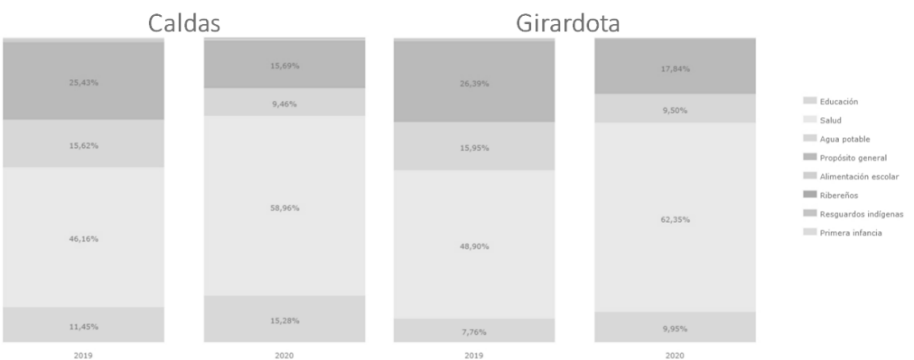

Nota: La asignación del SGP para Caldas es de \$9.236.345.103 y para Girardota es de \$ 8.437.419.731. La gráfica y valores son tomados de la página Terridata del DNP, 2020 https://terridata.dnp.gov.co/

En los dos municipios estudiados no son claros los mecanismos de financiación para lograr el 100\% de las metas sus proyectos y ODS.

Como referente se puede revisar los acuerdos municipales en materia tributaria del municipio de Cota en Cundinamarca, la cual hace uso de las atribuciones que le confiere la constitución política y la ley. Ver capítulo de Exenciones tributarias. Este municipio les ha ofrecido a las empresas que se trasladen a sus áreas urbanas o rurales o parques industriales y tecnológicos, beneficios tributarios tales como exenciones en el impuesto de Industria y Comercio durante los primeros diez años o pactando una tarifa reducida de este y, descuento, para estas y las ya existentes, de otros impuestos municipales a partir de la generación de empleo para los habitantes del municipio, esto se refleja hoy en sus ingresos que como los 
documenta Conde Finquitiva (2015), los ingresos corrientes para el año 2011 fueron de 57.844 millones y de estos 30.000 millones corresponden al rubro de Industria y Comercio. Estos ingresos han venido incrementando, y es así como en el año 2013 ya este rubro alcanzó los 40.300 millones.

Caracterización de las empresas

De acuerdo con las entrevistas realizadas a estas dos empresas en la Tabla 3 se observa la información relevante.

Tabla 3. Principales características del centro de Distribución

\begin{tabular}{|c|c|c|}
\hline Descripción & $\begin{array}{l}\text { Empresa de } \\
\text { Alimentos }\end{array}$ & Empresa de Aseo \\
\hline Municipio De Ubicación & Caldas & Girardota \\
\hline Área aproximada de las instalaciones en mts2. & 2.500 & 30.000 \\
\hline Año de inicio de Operaciones en esta Sede & 2016 & 2008 \\
\hline Cantidad de empleados totales de la sede & 28 & 110 \\
\hline $\begin{array}{c}\text { Porcentaje de Mano de obra Local (del Municipio donde se } \\
\text { encuentra la sede) }\end{array}$ & $10 \%$ & $80 \%$ \\
\hline \multicolumn{2}{|l|}{ Estándares de calidad que maneja } & BASC, ISO9001 \\
\hline \multirow{2}{*}{\multicolumn{3}{|c|}{$\begin{array}{l}\text { Nota: Respecto a la empresa dedicada a la producción de artículos de aseo y cuidado perso } \\
\text { anteriormente se encontraba ubicada en Sabaneta - Antioquia, municipio al sur del Valle de Abur } \\
\text { alimentos en el municipio de Itagüí, también al Sur del Valle de Aburrá. } \\
\text { La Tabla } 4 \text { recopila los servicios logísticos y tecnológicos que p } \\
\text { estos CEDIS. } \\
\text { Tabla 4. Servicios Logísticos y Tecnológicos implementados en los CEDIS }\end{array}$}} \\
\hline & & \\
\hline Descripción & $\begin{array}{l}\text { Empresa de } \\
\text { Alimentos }\end{array}$ & Empresa de Aseo \\
\hline Picking & $x$ & $x$ \\
\hline Packing & $x$ & $x$ \\
\hline CrossDocking & $x$ & $x$ \\
\hline Almacenamiento de Producto terminado & $x$ & $x$ \\
\hline Almacenamiento de Materia Prima & $x$ & \\
\hline Despacho Local & $x$ & $x$ \\
\hline Despacho Nacional & & $x$ \\
\hline Despacho Internacional & & $x$ \\
\hline
\end{tabular}




\begin{tabular}{ccc}
$\begin{array}{c}\text { Maquila de re-empaque } \\
\text { Seguimiento de vehículos/carga por medios electrónicos } \\
\text { ¿Hay cadena de frio? }\end{array}$ & $\mathrm{X}$ \\
\hline \multicolumn{1}{c}{ Servicios Tecnológicos } & $\mathrm{Si}$ \\
\hline WMS (warehouse management System) & $\mathrm{X}$ & $\mathrm{X}$ \\
RFID & & $\mathrm{X}$ \\
Código de barras & $\mathrm{X}$ \\
QR & $\mathrm{X}$ \\
\hline Voice picking & \\
\hline
\end{tabular}

Nota: Ninguna de las dos empresas tiene procesos productivos dentro de sus instalaciones.

Las actividades desarrolladas en la empresa del sector de alimentos solo tienen cobertura local, mientas que la ubicada en Girardota realiza distribución nacional e internacional.

La Tabla 5 recoge las motivaciones que se tuvieron en cuenta para la elección de la ubicación del centro de distribución

La ubicación del CEDIS de la zona norte se determinó a partir de un modelo de simulación para evaluar el lugar del área metropolitana al que se podrían desplazar. El centro de Medellín no resultó rentable a largo plazo puesto que el sector está superpoblado y no tenían espacio suficiente para ubicar las bodegas; además de las restricciones del POT. Dentro de las consideraciones se incluyeron las zonas céntricas, industriales y parques logísticos de los diferentes municipios del área metropolitana, para verificar tiempos de recorrido, movilidad, acceso a troncales viales, valor del metro cuadrado de tierras, beneficios tributarios, entre otros; concluyendo, a partir del software de simulación, que el lugar más adecuado era el Parque Logístico del municipio de Girardota. En 2008 se establecieron allí contando con una capacidad inicial de $18.000 \mathrm{mts}^{2}$ (actualmente tienen $30.000 \mathrm{mts}^{2}$ de terreno, de los cuales $20.000 \mathrm{mts}^{2}$ son para almacenaje de producto terminado). 
En cuanto a las motivaciones por las cuales las empresas decidieron reubicar sus CEDIS, estas se documentan en la Tabla 5

Tabla 5 Motivaciones para la reubicación del CEDI

\begin{tabular}{|c|c|c|}
\hline Descripción & $\begin{array}{l}\text { Empresa de } \\
\text { Alimentos }\end{array}$ & $\begin{array}{l}\text { Empresa de } \\
\text { Aseo }\end{array}$ \\
\hline Restricciones por planes de ordenamiento territorial & $\mathrm{x}$ & $\mathrm{x}$ \\
\hline Crecimiento y aumento de la capacidad de almacenamiento & $x$ & $x$ \\
\hline Optimización del espacio de almacenamiento & $x$ & \\
\hline \multicolumn{3}{|l|}{ Reubicación de toda la empresa } \\
\hline \multicolumn{3}{|l|}{ Finalización de contrato de arrendamiento } \\
\hline Necesidad de expansión & & $x$ \\
\hline \multicolumn{3}{|l|}{ Necesidad de consolidación de procesos } \\
\hline $\begin{array}{l}\text { Necesidad de Modernización de la planta de producción y/o } \\
\text { Almacenamiento }\end{array}$ & $x$ & $x$ \\
\hline $\begin{array}{l}\text { Disminución de costos logísticos (Transporte, Peajes consolidación de } \\
\qquad S K U s \text { ) }\end{array}$ & & $\mathrm{x}$ \\
\hline \multicolumn{3}{|l|}{ Cubrimiento de Nuevos mercados } \\
\hline Incremento en la demanda & $\mathrm{x}$ & \\
\hline \multicolumn{3}{|l|}{ Aprovechamiento de beneficios tributarios del municipio } \\
\hline \multicolumn{3}{|l|}{ Presiones medioambientales } \\
\hline Valor del metro cuadrado de tierra frente a otros municipios & & $\mathrm{x}$ \\
\hline Ubicación estratégica por vías y peajes & & $x$ \\
\hline Tiempos de recorrido & & $x$ \\
\hline
\end{tabular}

Nota: Las dos empresas afirmaron que los municipios elegidos no tienen exenciones o beneficios tributarios para los parques logísticos o para ellas dada su ubicación.

Respecto de la compañía del sector de alimentos, la cual se encuentra ubicada en el municipio de Caldas, destacaron que en la motivación principal para ubicarse en las afueras de la ciudad tuvo que ver el factor de espacio y las restricciones por POT ya que dentro de la ciudad resultaba complicado acceder a nuevos terrenos que pudieran adaptarse al incremento de la demanda, e igualmente el factor del transporte para el ingreso y salida de camiones con carga pesada.

En cuanto a los beneficios percibidos por las organizaciones, la Tabla 6 documenta los principales.

Tabla 6 Beneficios percibidos por la organización en la reubicación del CEDI 


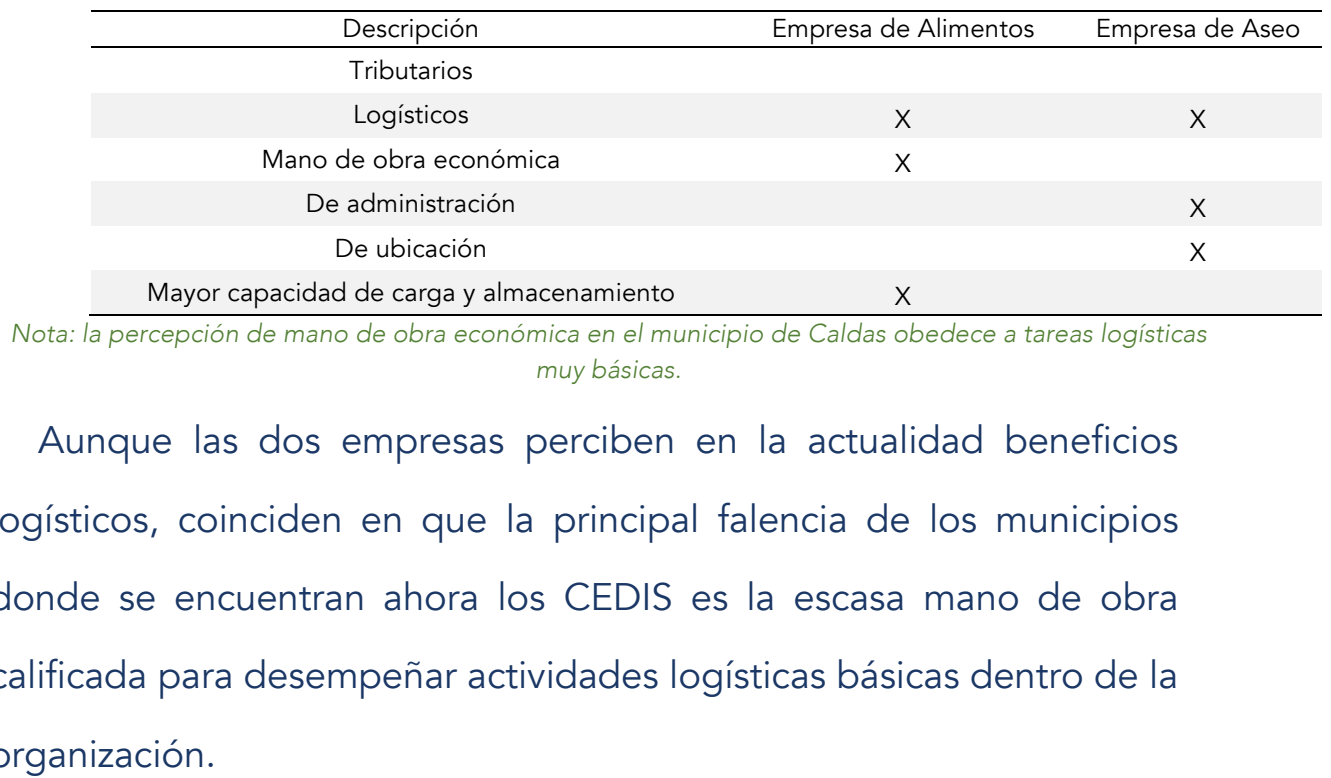

Aunque el número de participación de trabajadores en la planta total en el municipio de Girardota es alto (80\%) el entrevistado afirmó que esta mano de obra realiza tareas muy básicas como maquilas en procesos de empaque. Los procesos logísticos tales como el voicepicking, que requieren interacción con computadoras, es realizada por trabajadores que se desplazan desde otros municipios.

Tabla 7 Beneficios generados al municipio por la reubicación del CEDI

\begin{tabular}{ccc}
\hline Descripción & Empresa de Alimentos & Empresa de Aseo \\
\hline Tributarios & & $\mathrm{X}$ \\
Sociales & $\mathrm{X}$ & $\mathrm{X}$ \\
Medioambientales & & \\
\hline
\end{tabular}

Nota: Las dos empresas coinciden en que los beneficios generados han impactado más en el sector social.

Las dificultades presentadas en la implementación y puesta en marcha de los CEDI estuvieron principalmente representadas por problemas de movilidad. Durante los primeros años de actividades logísticas las vías de acceso no habían sido diseñadas para el tráfico de está y las demás instaladas en el parque, de tal suerte que si había 
algún incidente de tráfico las operaciones logísticas quedaban suspendidas mientras la autoridad local hacía los informes respectivos para dar pasó nuevamente a los vehículos automotores; sobre todo cuando se trataba de accidentes con consecuencias lamentables en la vida de peatones o pasajeros de los vehículos.

Adicionalmente, y a pesar de ser un parque configurado con todos los servicios, el fluido eléctrico influye en el normal desarrollo de las operaciones logísticas puesto que sus constantes caídas afectan en forma negativa su desempeño.

Por último, frente a las expectativas económicas que se tenían para el parque industrial de Girardota, cuando se realizaron los estudios no estaba dentro de los planes la instalación del peaje social en la frontera entre el municipio de Bello y Copacabana, lo que ha afectado en los resultados económicos esperados.

Las dos empresas enfatizan el impacto social positivo a los municipios donde se encuentra el CEDI puesto que hay pago de impuestos, generación de empleo y campañas y donaciones de los productos que fabrican.

\section{Conclusiones}

El desplazamiento de los centros de distribución logística hacia Parques Industriales y Logísticos por fuera de las áreas urbanas seguirá siendo tendencia gracias a los fenómenos ya descritos como los POT. la urbanización y el desplazamiento rural demográfico que obligan a 
repensar los planes básicos de ordenamiento territorial de ciudades y municipios y alinearlos buscando dar cumplimiento a los ODS.

Entre las ventajas y beneficios que trae estar en un parque industrial se incluyen las economías de escala, puesto que el parque puede conformar grupos de empresas del mismo sector que pueden ejercer presiones de negociación por compras a gran escala. En temas de producción, pueden usar tecnologías más limpias puesto que con la instalación en espacios nuevos, se incluyen procesos productivos modernos cuyas tendencias son ecoeficientes.

Es necesario que las finanzas públicas instruyan a candidatos para alcaldías y gobernaciones en la elaboración de Planes de Desarrollo, a partir del aprovechamiento de las atribuciones de ley para administrar los impuestos municipales, que permitan obtener fondos para financiar los proyectos de ODS.

En cuanto a los ODS regionales, a partir de la hoja de ruta trazada, aún es muy temprano para evaluar el cumplimiento de metas, cuando todavía se encuentran algunos de estos en etapa de construcción.

Las limitaciones y tipo de investigación permiten inferir resultados que se podrían confirmar más profundamente con la inclusión de otras empresas que ya se han desplazado a parques industriales de los municipios revisados y de otros existentes en el Valle de Aburrá.

La temática permite futuras investigaciones en las que se puedan profundizar en las relaciones de otros ODS con los parques logísticos y de la incidencia real con el $\mathrm{N}^{\circ} 9$, puesto que como se mencionó anteriormente, en Colombia aún es muy temprano para cuantificar resultados reales. 


\section{Referencias}

Acuerdo N 48 de 2014. "Por medio del cual se adopta la revisión y ajuste de largo plazo del Plan de Ordenamiento Territorial del Municipio de Medellín y se dictan otras disposiciones complementarias". Diciembre 17 de 2014. Gaceta Oficial Nº 4267. Municipio de Medellín.

Acuerdo Metropolitano No31 de 2019. . "Por medio del cual se adopta el Plan Estratégico Metropolitano de Ordenamiento Territorial del Valle de Aburrá". Diciembre 19 de 2019. Gaceta Oficial N4656. Municipio de Medellín.

Cámara de Comercio de Medellín - [CCM]. (2019). Cómo avanza la Economía de Antioquia 2019

https://www.camaramedellin.com.co/Portals/0/conozca_la_camara/informesSIC/Informe-Economico-SIC2019.pdf

Carlino, H., Netto, M., Suarez, E., \& Vasa, A. (2017). La contribución de la banca pública de desarrollo a los Objetivos de Desarrollo Sostenible en los países de América Latina y el Caribe (BID (ed.); IDB-DP-499). https://publications.iadb.org/publications/spanish/document/La-contribución-de-labanca-pública-de-desarrollo-a-los-Objetivos-de-Desarrollo-Sostenible-en-los-paísesde-América-Latina-y-el-Caribe.pdf

Conde-Fiquitiva, M.-I. (2015). Impacto Socioeconómico de los Beneficios y Exenciones Tributarias para las Empresas registradas en la Zona Industrial del Municipio de Cota Cundinamarca. (pp. $1-26)$. https://repository.unimilitar.edu.co/bitstream/handle/10654/11469/Ensayo\%20UMNG $\% 20-$

\%20Mar\%EDa\%20In\%E9s\%20Conde.pdf;jsessionid=E8387A6648679528A7D1E8F01 E345490?sequence $=1$

Consejo Municipal de Caldas (2020). Plan de Desarrollo para la Transformación Social, Económica y Ambiental del Municipio de Caldas 2020-2023. https://www.caldasantioquia.gov.co/pd/

Consejo Municipal de Girardota. (2019). Plan De Desarrollo Territorial 2020-2023: Girardota, Antioquia. http://www.girardota.gov.co/NuestraAlcaldia/SaladePrensa/Paginas/AhoraS\%C3\%AD-Girardota-para-todos-Plan-de-Desarrollo-Territorial-2020-\%E2\%80\%932023.aspx

Constitución Política de Colombia [Const]. Art. 294. 7 de julio de 1991 (Colombia).

Departamento Nacional de Planeación - [DNP]. (2008). CONPES 3547 Política Nacional Logística. http://wsp.presidencia.gov.co/sncei/politica/Documents/Conpes-354727oct2008.pdf

DNP. (2015). Desempeño Fiscal de los Departamentos y Municipios. https://colaboracion.dnp.gov.co/CDT/Desarrollo Territorial/Desempeño Fiscal 2015 V.2016.11.09.pdf 
DNP. (2018). Conpes 3918 Estrategia Para La Implementación De Los Objetivos De Desarrollo Sostenible (ODS) En Colombia. In Documento Conpes 3918. https://colaboracion.dnp.gov.co/CDT/Conpes/Económicos/3918.pdf

DNP. (2019). Compes 3975: Política nacional para la transformación digital e inteligencia artificial. In Consejo Nacional de Politica Economica y Social - Republica de Colombia. https://www.mintic.gov.co/portal/604/articles-107147_recurso_1.pdf

DNP, Departamento Nacional de Planeación -. (2020). CONPES 3982 Política Nacional Logística. https://colaboracion.dnp.gov.co/CDT/Conpes/Económicos/3982.pdf

DNP, Directorio Nacional de Planeación -. (1995). CONPES 2791 -Ajustes al Sistema Nacional de

Cofinanciación-. https://colaboracion.dnp.gov.co/CDT/CONPES/Econ\%C3\%B3micos/2791.pdf

Division de Impuestos y Aduanas Nacionales [DIAN]. (2000). Ley Páez y Ley Quimbaya, Tratamientos Tributarios Preferenciales (No. 011-054). https://www.dian.gov.co/dian/cifras/Otros Cuadernos de Trabajo/054. Ley Páez y Ley Quimbaya.pdf

Instituto para el Desarrollo de Antioquia - IDEA. (2014). Guía práctica para la elaboración, presentación y ejecución del presupuesto municipal (17th ed.). https://www.idea.gov.co/BibliotecaPublicaciones/Guía práctica para la elaboración, presentación y ejecución del presupuesto municipal.pdf

Leal, J. (2005). Ecoeficiencia: marco de análisis, indicadores y experiencias. In C. de Miguel \& J.-J. Gómez. (Eds.), Publicación de las Naciones Unidas: Vol. Vol. 1 (Issue No. 1). Naciones Unidas. http://www.cepal.org/es/publicaciones/5644-ecoeficiencia-marcode-analisis-indicadores-y-experiencias

Ley 14 de 1983. Por la cual se fortalecen los fiscos de las entidades territoriales y dictan otras disposiciones. 6 de Julio de1983. D. O. No. 36.288

Ley 788 de 2002. Por la cual se expiden normas en materia tributaria y penal del orden nacional y territorial; y se dictan otras disposiciones. 27 de diciembre de 2002. D. O. No. 45.046

Ley 1900 de 2018. Por medio de la cual se establecen criterios de equidad de géneros en la adjudicación de las tierras baldías, vivienda rural, proyectos productivos, se modifica la ley 160 de 1994 y se dictan otras disposiciones. 18 de Junio de 2018. D. O. 1.

Majchrzak-Lepczyk, J. (2017). Shaping the Warehouse Space Market Countries Central and Eastern Europe. Research in Logistics and Production, 7(2), 103-112. https://doi.org/10.21008/j.2083-4950.2017.7.2.4

McKinnon, A. (2009). The present and future land requirements of logistical activities. Land Use Policy, 26(SUPPL. 1), 293-301. https://doi.org/10.1016/j.landusepol.2009.08.014

Monroy, N., \& Ramírez, D. (2003). Parques Industriales Ecoeficientes en Bogotá: ¿Una Alternativa Ambiental, Económica y/o Social? Revista de Ingeniería, 0(19), 126-138. https://doi.org/10.16924/riua.v0i19.447

Notteboom, T. E., \& Rodrigue, J. P. (2005). Port regionalization: Towards a new phase in port development. Maritime Policy and Management:The Flagship Journal of International Shipping and Port Research, 32(3), 297-313. https://doi.org/10.1080/03088830500139885 
Organizacion de las Naciones Unidas [ONU]. (1992). UN Conference on Environment and $\begin{array}{lllll}\text { Development. In } & \text { Agenda June). }\end{array}$ https://doi.org/10.4135/9781412971867.n128

ONU. (2002). Johannesburg Plan of Implementation: Plan of Implementation of the World Summit on Sustainable Development Contents. Johaneesburg Plan of Implementation, $1-62$.

ONU. (2015a). Addis Ababa Action Agenda of the Third International Conference on Financing for Development. In United Nations Department of Economic and Social Affairs. Financing for Development Office. http://www.un.org/esa/ffd/wpcontent/uploads/2015/08/AAAA_Outcome.pdf

ONU. (2015b). Resolución A/Res/69/313 aprobada por la Asamblea General (Vol. 12674). https://www.un.org/es/ga/69/resolutions.shtml

ONU. (2016). 9 Industria, Innovación e Infraestructura. Objetivos de Desarrollo Sostenible. https://www.un.org/sustainabledevelopment/es/infrastructure/

ONU. (2019). International Guidelines For Industrial Parks. In Inclusive and Sustainable Industrial Development. https://www.unido.org/sites/default/files/files/201911/International_Guidelines_for_Industrial_Parks.pdf

Phillimore, J., \& Joseph, R. (2003). Science Parks: A Triumph of Hype over Experience? In The International Handbook on Innovation. Elsevier Ltd. https://doi.org/10.1016/B978008044198-6/50051-6

Ramírez, J.-M., \& Higuera, L. (2018). Cambio Estructural y Desarrollo Sostenible en Colombia. In Políticas industriales y tecnológicas en América Latina (Vol. 2, Issue 7, pp. 277-314). CEPAL. https://doi.org/10.37228/estado_comunes.v2.n7.2018.95

Rojas B, O. (2015). Situación de Tierras en Colombia: marco normativo. https://www.porlatierra.org/docs/7e1ee0b91117c3f61dc12f5f0b9f8052.pdf

Sánchez-Cubides, P.-A. (2014). Estructura de los ingresos territoriales en Colombia. Criterio Jurídico Garantista, 6(11), 160-175. http://revistas.fuac.edu.co/index.php/criteriojuridicogarantista/article/view/445/429

Secretaría Técnica de la Comisión ODS. (2018). Reporte nacional voluntario-Colombia Objetivos de Desarrollo Sostenible. www.dnp.gov.co

Vidova, J. (2010). Industrial parks-history, their present and influence on employment. Review of Economic Perspectives, X(1), 41-58. https://doi.org/10.2478/v10135-009-0008-1

Wilmsmeier, G. (2015). Geografía del transporte de carga: evolución y desafíos en un contexto global cambiante. In CEPAL, Naciones Unidas. http://repositorio.cepal.org/handle/11362/39660 